\title{
Whole-exome sequencing reveals a rare missense variant in DTNA in an Iranian pedigree with early-onset atrial fibrillation
}

\author{
Mahshid Malakootian', Masoumeh Jalilian', Samira Kalayinia' ', Maryam Hosseini Moghadam', \\ Mona Heidarali and Majid Haghjoo ${ }^{2^{*}}$
}

\begin{abstract}
Atrial fibrillation (AF) is a morbid and heritable irregular cardiac rhythm that affects about $2 \%-3 \%$ of the population. Patients with early-onset AF have a strong genetic association with the disease; nonetheless, the exact underlying mechanisms need clarification. We herein present our evaluation of a 2-generation Iranian pedigree with early-onset AF. Whole-exome sequencing was applied to elucidate the genetic predisposition. Direct DNA sequencing was utilized to confirm and screen the variants in the proband and his available family members. The pathogenicity of the identified nucleotide variations was scrutinized via either segregation analysis in the family or in silico predictive software. The comprehensive variant analysis revealed a missense variant (c.G681C, p.E227D, rs 1477078144) in the human a-dystrobrevin gene (DTNA), which is rare in genetic databases. Most in silico analyses have predicted this variant as a disease-causing variant, and the variant is co-segregated with the disease phenotype in the family. Previous studies have demonstrated the association between the DTNA gene and left ventricular noncompaction cardiomyopathy. Taken together, we provide the first evidence of an association between a nucleotide variation in the DTNA gene and early-onset AF in an Iranian family. However, the genetic testing of AF in the Iranian population is still limited. This finding not only further confirms the significant role of genetics in the incidence of early-onset AF but also expands the spectrum of the gene variations that lead to AF. Additionally, it may have further implications for the treatment and prevention of AF.
\end{abstract}

Keywords: Early-onset AF, DTNA, Genetic testing, Cardiac arrhythmia

\section{Introduction}

Atrial fibrillation (AF) is the most common irregular cardiac rhythm in clinical practice, such that its incidence and prevalence have come to the dimension of a twenty first-century cardiovascular disorder epidemic $[1,2]$. Several risk factors such as age, sex, obesity, alcohol, diabetes mellitus, valvular heart diseases, and hypertension

\footnotetext{
*Correspondence: majid.haghjoo@gmail.com

${ }^{2}$ Cardiac Electrophysiology Center, Rajaie Cardiovascular Medical and Research Center, Iran University of Medical Sciences, Vali-Asr St, Hashemi-Rafsanjani Blvd, Tehran, Iran

Full list of author information is available at the end of the article
}

are correlated with the incidence of AF [3]. Nonetheless, about $30 \%$ of patients with AF present with a normal heart and without any association of known cardiac pathologies or conventional risk factors [4]

Mounting evidence indicates the role of genetics as important predisposing factors in the development of AF [5, 6]. Additionally, having a positive family history increases the risk of developing early-onset AF [7-11]. In familial cases, nearly 1 in 4 patients has a first-degree relative who suffers from AF [12]. According to the Iranian Registry of Atrial Fibrillation, history of AF in a first-degree relative is positive in $15.3 \%$ of the Iranian AF population $[7,13]$. 
Thus far, research has identified more than 100 different loci and 160 genes in association with $\operatorname{AF}[6,14]$. Furthermore, mutations in cardiac ion channels (viz, potassium, sodium, and calcium) and non-ion channel coding genes such as signaling molecules, myocardial structural proteins, and cardiac transcription factors have been linked with familial or early-onset AF [15-23].

In the present study, we investigated the genetic predisposition of a 2-generation pedigree with early-onset AF by performing whole-exome sequencing (WES), followed by direct Sanger sequencing. Herein, we report the identification of a novel mutation in the human $\alpha$-dystrobrevin gene (DTNA), which may have caused the AF phenotype in the family.

\section{Material and methods}

\section{Subjects}

The present study enrolled 7 members of 1 family. Some of them clinically presented early-onset AF (age $\leq 65 \mathrm{y}$ ), while the others had no evidence of underlying structural or systemic diseases. The study protocol was approved by the National Institute for Medical Research Development (NIMAD, 971510) and the Ethics Committee of Rajaie Cardiovascular Medical and Research Center (RHC.AC.IR.REC.1397.088). The study was conducted in accordance with the Helsinki Declaration. All the individuals who joined the study signed written informed consent.

\section{DNA extraction and WES}

Genomic DNAs were isolated from $200 \mu \mathrm{L}$ of peripheral blood samples of all available family members in the pedigree utilizing a DNA extraction kit (DNPTM Kit, Iran). For WES, the SOLIDv4 platform (SureSelect X Kit, Macrogen South Korea) was applied according to the manufacturer's instructions. Sequences acquired from WES were aligned to the GRCh37/hg19 human reference genome. Then, the WES-extracted variants were filtered, and bioinformatics software platforms PolyPhen (http:// genetics.bwh.harvard.edu/pph2/), SIFT (https://sift.bii.astar.edu.sg/), PROVEAN (http://provean.jcvi.org/index. php), and MutationTaster (http://www.mutationtaster. org/) were applied to predict the pathogenicity of the variants. The variants were classified in keeping with the guidelines of the American College of Medical Genetics and Genomics (ACMG) [24].

\section{Polymerase chain reaction (PCR), primer design, and Sanger sequencing}

The nucleotide variations detected by WES were confirmed by Sanger sequencing with a 3500 Genetic analyzer (Applied Biosystems, USA) in the proband and evaluated in all the individuals in the family.
Concisely, specific oligonucleotides (oligos) were designed to amplify a fragment that covered the candidate variants in the suggested genes. The sequences of all the primers are presented in Additional file 1: Table S1.

The PCR condition for amplifying the region was as follows: initial denaturation at $94{ }^{\circ} \mathrm{C}$ for $5 \mathrm{~min}$, followed by 30 cycles at $94{ }^{\circ} \mathrm{C}$ for $30 \mathrm{~s}, 61^{\circ} \mathrm{C}$ for $30 \mathrm{~s}[\alpha$-dystrobrevin (DTNA)], $59^{\circ} \mathrm{C}$ for $30 \mathrm{~s}$ [Nebulette (NEBL)], $60^{\circ} \mathrm{C}$ for $30 \mathrm{~s}$ [Sodium Voltage-Gated Channel a Subunit 5 (SCN5A)], and $72{ }^{\circ} \mathrm{C}$ for $30 \mathrm{~s}$, with a final extension at $72{ }^{\circ} \mathrm{C}$ for $10 \mathrm{~min}$. The products were electrophoresed on a $1 \%$ agarose gel, stained with ethidium bromide, and visualized under ultraviolet light. Forward or reverse primers were utilized to sequence the parts of interest.

\section{Bioinformatics analysis}

The Gene Runner (Gene Runner 6.5.50) and PerlPrimer (PerlPrimer 1.1.21) software tools were utilized to design the primers. For the analysis of the sequencing results, the BioEdit software (BioEdit 7.2.1) was applied. The identified nucleotide transitions were investigated through the UCSC Genome Browser (https://genome. ucsc.edu) and ClinVar (www.ncbi.nlm.nih.gov/clinvar) databases. Further, in silico predictive software tools such as SIFT (https://sift.bii.a-star.edu.sg), PROVEAN (provean.jcvi.org), PolyPhen-2 (genetics.bwh.harvard. edu/pph2), and MutationTaster (www.mutationtaster. org) were applied to study the pathogenesis of the detected nucleotide variations.

The Protein Homology/analogY Recognition Engine V 2.0 (Phyre2) [25] and DNASTAR (https://www.dnastar. com) bioinformatics software tools were utilized to predict the secondary and third structures of the DTNA wild type and mutant protein.

\section{Results}

\section{Clinical phenotype of the family}

The family of interest was a 2-generation pedigree suffering from AF and heart diseases (Fig. 1).

The proband in the family (III-5) was a 47-year-old man, who was admitted to Rajaie Cardiovascular Medical and Research Center with suspected AF. For the first time, AF was diagnosed during the evaluation of plasmacytomas. He was asymptomatic. Electrocardiography showed AF with a rapid ventricular rate (Fig. 2). The AF rhythm was observed before the commencement of chemotherapy. Transthoracic echocardiography showed a decreased left ventricular function (ejection fraction $=25 \%$ ), a normal left ventricular size, mild left atrial enlargement, mild-to-moderate mitral regurgitation, no left ventricular hypertrophy, and no noncompaction pattern. No evidence of late enhancement or left ventricular noncompaction was observed in cardiac magnetic 


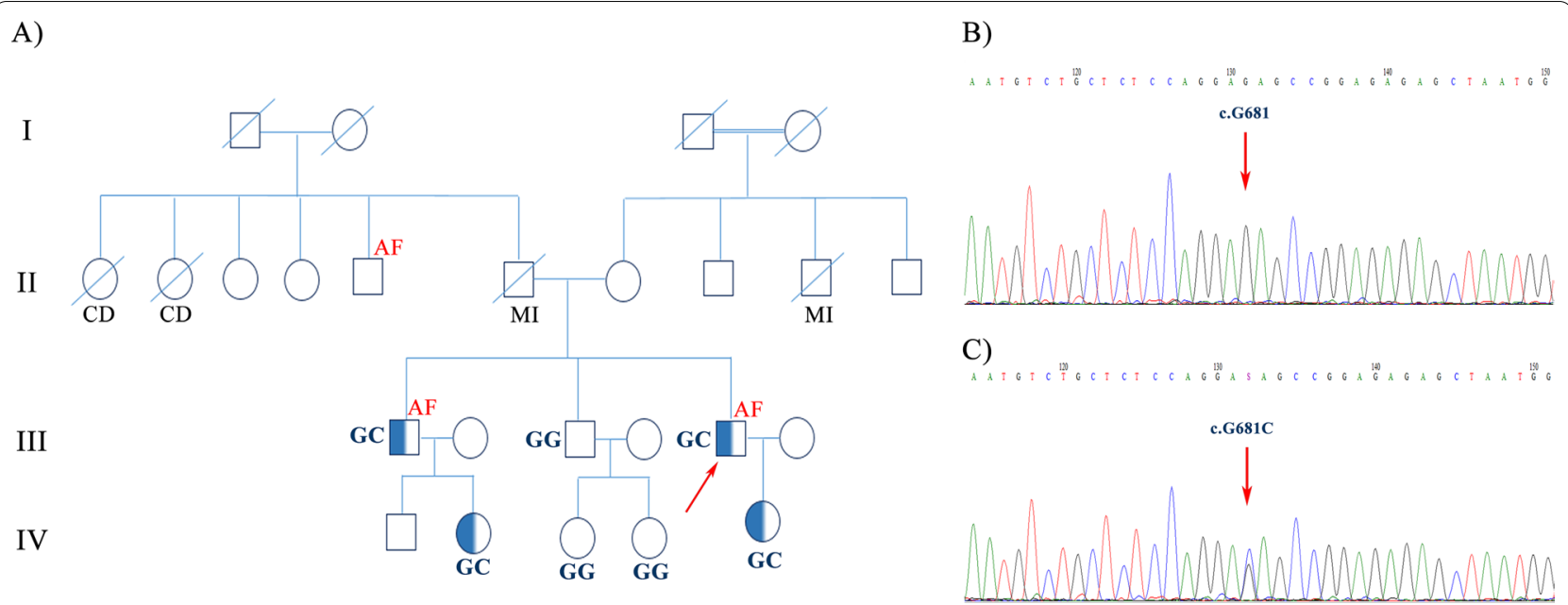

Fig. 1 The image illustrates the pedigree, sequencing analysis, and chromatogram of the index family carrying the mutation, c.G681C, in the DTNA gene. A The pedigree of the proband revealed a positive family history of AF and MI. The genotype of the proband and his family members for DTNA transition demonstrated that the proband (III-5), his brother (III-1), and his brother's children (IV-2 and IV-5) carried the c.G681C mutation of the DTNA gene in a heterozygote form. The other brother (III-3) and his children (IV-3 and IV-4), who were clinically normal, carried the wild type (G) of the nucleotide. $\mathbf{B}$ and $\mathbf{C}$ The chromatogram of the wild type (homozygote) and the mutant (heterozygote) of the identified transition is depicted respectively in the upper panel and the lower panel. AF: Atrial Fibrillation, MI: Myocardial Infarction

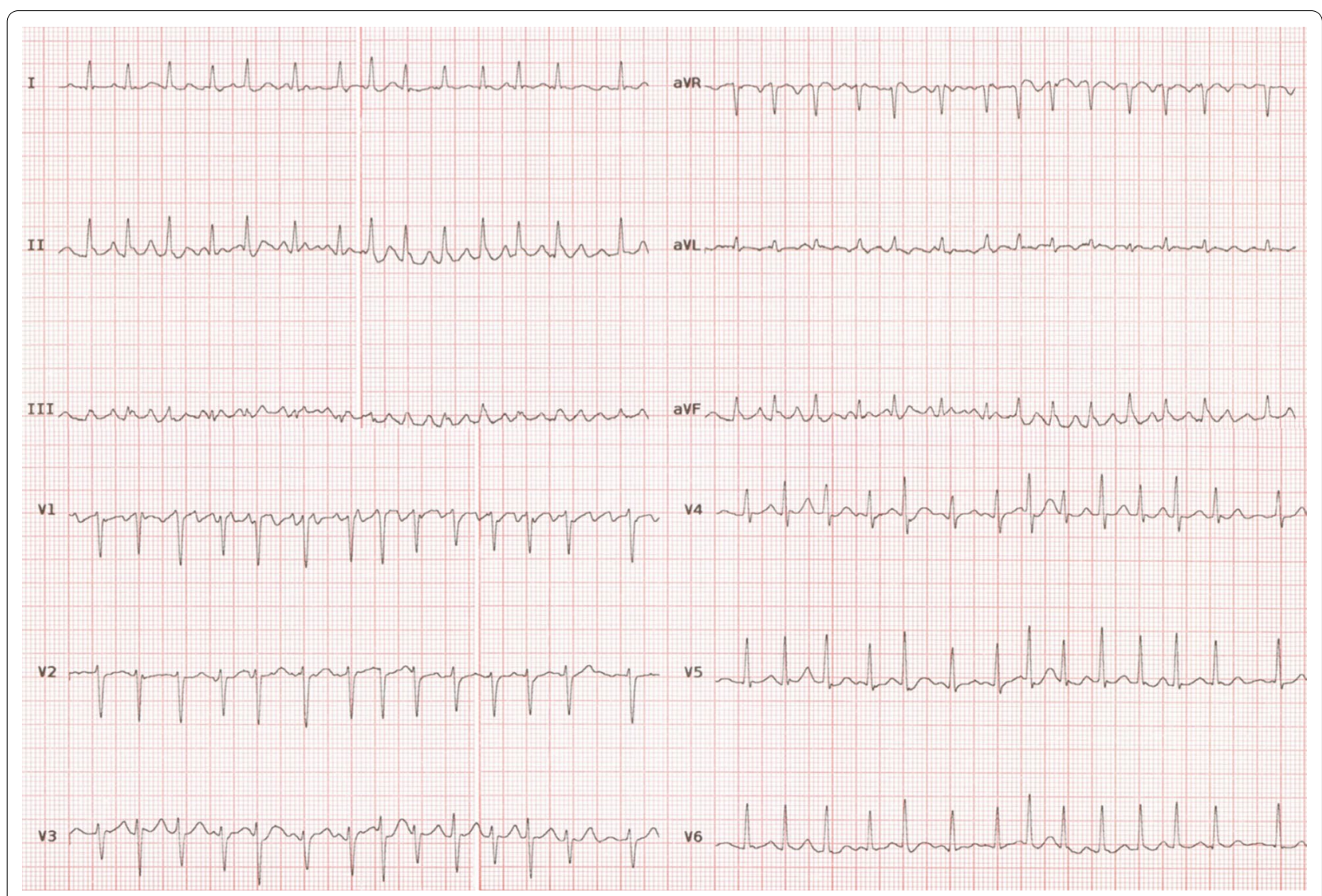

Fig. 2 ECG of proband (III-5) shows narrow QRS tachycardia with irregular ventricular response and fibrillatory atrial activity 
resonance imaging. AF was initially converted successfully into the sinus rhythm with direct-current cardioversion. Electrocardiography after the cardioversion showed a normal sinus rhythm with no evidence of left atrial abnormality or left ventricular hypertrophy. After 3 months, AF episodes returned. Therefore, cryoballoon pulmonary vein isolation was done, which was successful. One month after the procedure, the left ventricular function showed a significant improvement (45-50\%) (Additional file 1: Table S2).

The proband's medical history was otherwise not significant for hypertension, diabetes, high cholesterol, and other cardiovascular diseases. His family history was positive for AF in one of his brothers (III-I) and his uncle (paternal family). There was a positive history of heart diseases from both maternal and paternal sides.

The proband's older brother (III-I) presented with drug-refractory paroxysmal $\mathrm{AF}$ at the age of 46 years for the first time. He also had a history of newly diagnosed systemic hypertension. Resting 12-lead electrocardiography showed a normal sinus rhythm and normal PR, QRS, and QTc intervals with no evidence of left atrial abnormality, left ventricular hypertrophy, or noncompaction patterns. Transthoracic echocardiography revealed a normal left ventricular function (55\%), normal left atrial dimensions, a good valvular function, and no evidence of left ventricular hypertrophy. He underwent successful percutaneous pulmonary vein isolation using a 3D mapping system and radiofrequency energy.

At the age of 50 years, he presented with new-onset typical angina pectoris. Coronary angiography showed single-vessel disease, for which he received medical therapy. His angina pectoris progressed over time, and repeat coronary angiography when he was 55 years of age showed triple-vessel disease, for which he received 3 coronary stents. Two years after the percutaneous coronary intervention, he experienced recurrent episodes of AF; consequently, cryoballoon pulmonary vein isolation was performed, which was successful. During a 1-year followup after the second pulmonary vein isolation, he was in a stable medical condition (Additional file 1: Table S2).

The other brother of the proband and the other members of the proband's family who were available for examinations were apparently normal (Additional file 1: Table S2).

\section{Genetic findings}

For the determination of the causative genetic alterations in the family, an unbiased next-generation DNA sequencing, which covered the entire coding exons (WES), was performed. WES was accomplished with a mean target coverage rate of $100 \mathrm{X}$. The results were successively analyzed with Bowtie, [26] Freebayes, [27] and SnpSift. Filtering was accomplished based on different projects (viz, db-SNP, gnomAD v2, and 1000 Genomes Project databases) and other available population databases (eg, Iranome).

Three candidate variants were submitted for co-segregation analysis, and only 1 survived (Table 1 ).

The c.G681C transition (rs1477078144) was positioned in a conserved part of exon 6 of the DTNA gene located on Chr 18q12.1 (the GRCh37/hg19), resulting in a Glu227-to-Asp substitution in the $\alpha$-helix structure of the protein. This variant is reported neither in the 1000G Project nor in the Iranome Database. The in silico analysis of this variant via PolyPhen, SIFT, MutationTaster, and PROVEAN predicted this variant as probably damaging, deleterious, disease-causing, and neutral, respectively.

Sanger sequencing of this variant was performed in the proband and all 6 family members to confirm the presence and pattern of inheritance (Fig. 1). The Sanger sequencing upshots demonstrated that the index patient (III-5) and his affected brother (III-1) carried the variant in a heterozygous status (GC), while the other unaffected brother (III-3) and his children (IV-3 and IV-4) carried the wild type of the variant (GG) (Fig. 1).

The proband's only child (IV-5, 16 years old) and one of the 2 children of the affected brother (IV-2, 23 years old) carried the probable pathogenic variant like their respective father in the heterozygous form (Table 2). Both children appeared normal.

The proband's uncle (II-5), aged 62 years, showed symptoms of AF at an earlier age, but he was not available for the present study (Fig. 1).

The second (c.T298C) and third (c.A1673G, rs1805124) variations were harbored in exon 3 and exon 12 of the NEBL and SCN1A genes, respectively. The c.T298C transition of the $N E B L$ gene caused a Ser100-to-Pro substitution, and the c.A1673G nucleotide change led to the replacement of histidine 558 with arginine of SCN1A. The c.T298C variant is reported neither in the 1000G Project nor in the Iranome Database. PolyPhen, SIFT, PROVEAN, and MutationTaster programs predicted this variant as disease-causing and deleterious. The Sanger sequencing of this variant demonstrated the heterozygote states of this variant in the proband and his son. Neither of his brothers (affected and healthy ones) and their children carried this variant (Table 2). The third candidate variant, c.A1673G, was harbored in the SCN1A gene and previously reported as pathogenic. The results of the direct sequencing of this variant showed the heterozygote form of this variant in the proband and all his examined family members (Table 2). 


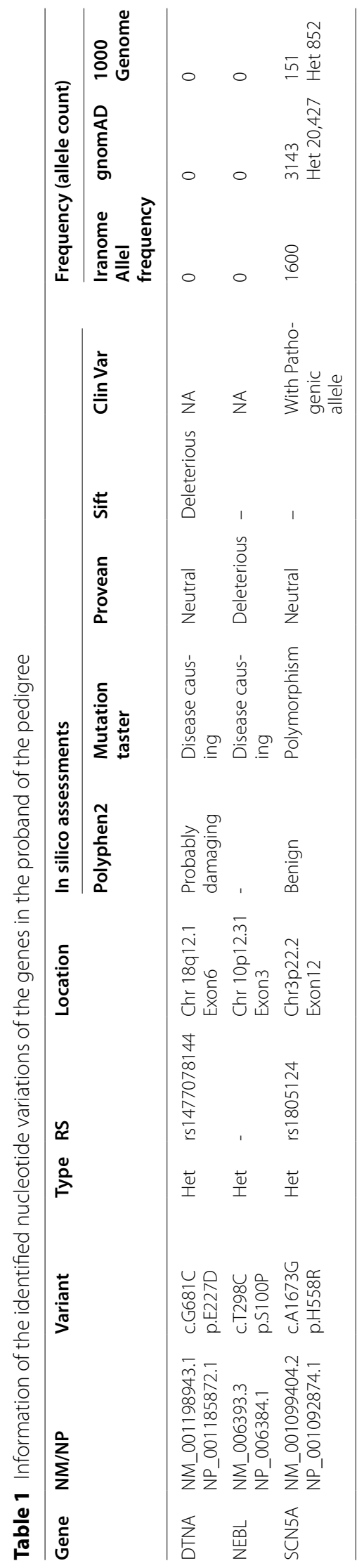


Table 2 Genotypes of all 3 identified variants in 7 available members of the family

\begin{tabular}{|c|c|c|c|}
\hline Individuals & DTNA(NM_001198943.1) (c.G681C) & NEBL(NM_006393.3) (c.T298C) & $\begin{array}{l}\text { SCN5A(NM_001099404.2) } \\
\text { (c.A1673G) }\end{array}$ \\
\hline|| $\mid-1$ & GC & $\mathrm{TT}$ & AG \\
\hline|| $\mid-3$ & GG & $\mathrm{TT}$ & AG \\
\hline|| $\mid-5$ & GC & $\mathrm{TC}$ & $A G$ \\
\hline $\mathrm{IV}-2$ & GC & $\mathrm{TT}$ & $A G$ \\
\hline IV-3 & GG & $\mathrm{TT}$ & AG \\
\hline IV-4 & GG & $\mathrm{TT}$ & AG \\
\hline IV-5 & GC & $\mathrm{TC}$ & $A G$ \\
\hline
\end{tabular}

\section{Discussion}

In recent decades, several approaches such as linkage analysis, candidate gene, and next-generation sequencing have revealed distinct disease-causing nucleotide variations in many genes linked to AF [14, 28]. Additionally, genome-wide association studies have introduced some loci associated with AF $[6,29]$. Although these findings have provided fresh insights into the underlying pathophysiology of AF and may clarify novel therapeutic pathways, the vast majority of the heritability of AF remains baffling.

In this study, we found a nucleotide transition, c.G681C, in the DTNA gene, which probably caused early-onset $\mathrm{AF}$ in an autosomal dominant inheritance pattern in an index Iranian family. To the best of our knowledge, the present study is the first investigation to report the point nucleotide variation in the DTNA gene associated with AF. This rare variant is not reported in the 1000G Project, the gnomAD Database, and the Iranome Database. In addition, most of the in silico software tools that we employed in the current investigation predicted this variation as probably damaging, deleterious, and disease-causing.

Notably, $\alpha$-dystrobrevin is related to dystrophin-related and dystrophin-associated proteins, which are thought to have a significant role in the stability and maintenance of the plasma membrane in the course of muscle contraction and relaxation, [30] while the exact function of dystrobrevin remains to be determined. A previous study reported that $\alpha$-dystrobrevin encoded by the DTNA gene made a scaffold unit structure at the sarcolemma of the heart muscle and was engaged in maintaining the structural integrity of muscle fibers [31]. Previous studies have demonstrated that mutations in $\alpha$-dystrobrevin lead to left ventricular noncompaction cardiomyopathy [31, 32] and Meniere's disease [33]. In 2001, Ichidea et al. reported a 362C-T transition (heterozygote form) in exon 3 of the DTNA, which substituted pro121-to-leu (P121L) at a protein level as a causative nucleotide alteration and resulted in left ventricular noncompaction in 6 affected members of a 4-generation Japanese family [32]. Further, a heterozygous mutation, c.146A-G, at a position of 146 resulting in an amino acid change from asparagine to serine at codon 49 was identified in a 39-year-old man with a diagnosis of left ventricular noncompaction cardiomyopathy [31]. The functional analysis of this variation confirmed the causal role of the DTNA-p.N49 S mutation in the pathogenesis of left ventricular noncompaction cardiomyopathy by generating a transgenic mouse model expressing the DtnaN49S mutant specifically in the heart [31]. The novel heterozygous missense variant was recognized in the DTNA gene (chr18: 32462094G.T), causing an amino acid change (valine to phenylalanine) in a patient with autosomal-dominant familial Meniere's disease [33]. Furthermore, 13 different variations of the DTNA gene in addition to the 2 aforementioned ones related to left ventricular noncompaction cardiomyopathy and Meniere's disease have been reported with conflicting interpretations of pathogenicity according to the ClinVar Database (Additional file 1: Table S3).

Our segregation analysis demonstrated that the proband and his affected brother (III-1), together with the their respective children (IV-2 and IV-5), carried the c.G681C transition (rs1477078144) in a heterozygote status in the DTNA gene, while the proband's unaffected brother (III-3) and his children (IV-3 and IV-4) did not carry the mutation and were normal based on clinical assessments as well. The children of the affected brothers in the family were apparently normal, which is probably due to their young age or the incomplete penetrance of the mutation. It is probable that the proband's uncle (paternal side), who also had AF, carried the mutation. Unfortunately, he was not available for further analysis.

Moreover, the whole DTNA, which is located in chromosome 18q12 and is composed of 23 coding exons, ranges in size from 9 to $214 \mathrm{bp}$ (Fig. 3). The largest open reading frame of dystrobrevin is in a transcript, $6.5 \mathrm{~kb}$ in length, termed "dystrobrevin-1", which is highly expressed in the brain and muscles. Other transcripts of $D T N A$ encode the functional isoforms of dystrobrevin 


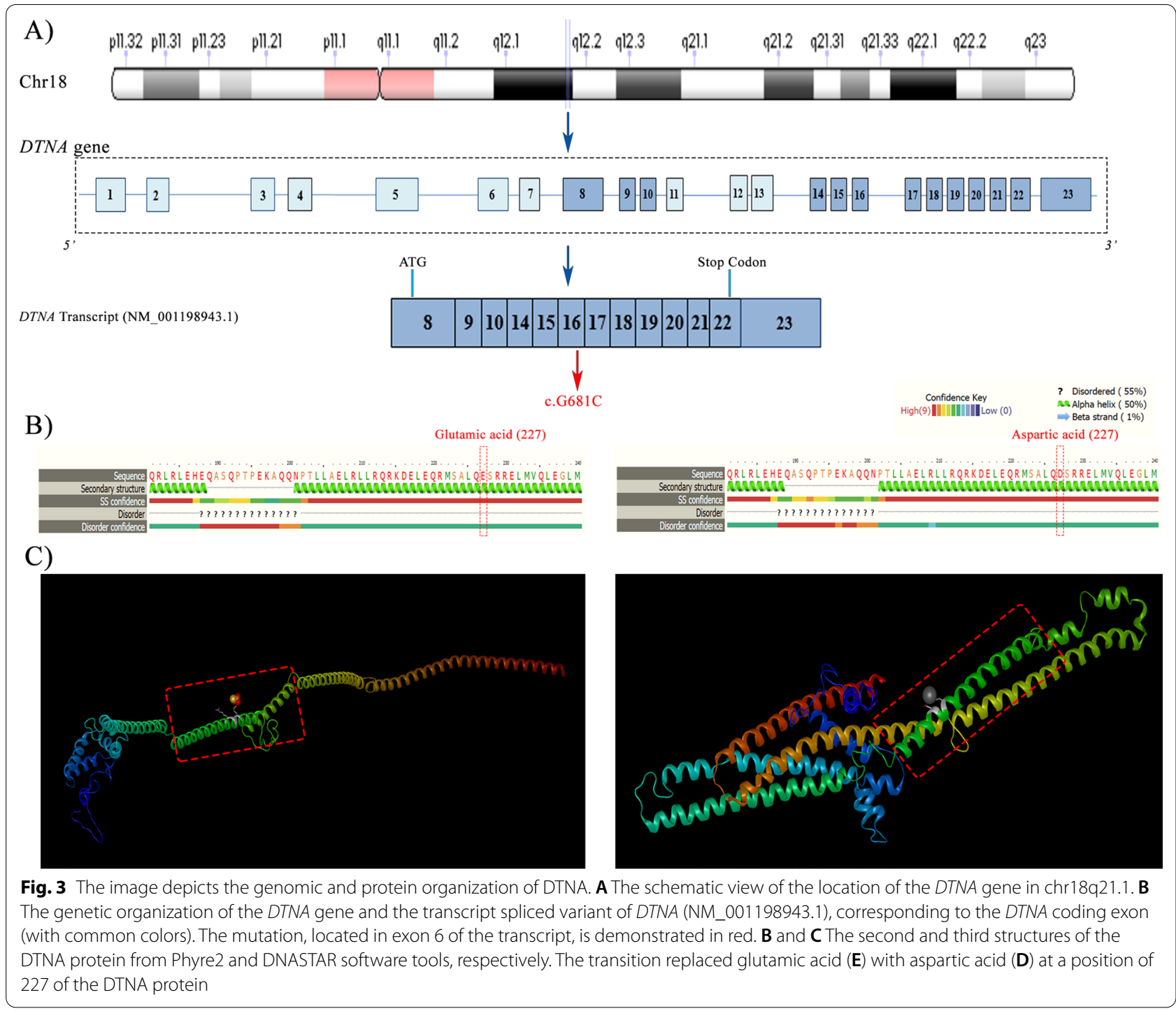

categorized as class I and class II [30]. The aforementioned isoforms are highly expressed in cardiac muscle as well as the brain and skeletal muscles. Class-I transcripts, characterized by alternatively spliced 3' ends, are predicted to create truncated protein isoforms. Class-II transcripts, characterized by a different 5 ' start site, are harbored in exon 8 of the DTNA gene [30,34]. The mutation that we identified in the present study (c.G681C, p.E227D, rs1477078144) is harbored in a transcript with NM-001198943.1 and belongs to the class-II transcripts of DTNA, which is highly expressed in cardiac muscle.

According to UniProt database (https://www.unipr ot.org/uniprot/Q9Y4J8\#interaction) (Q9Y4J8), DTNA interacts with Alpha-catulin (CTNNAL1) which is modulate the Rho pathway signaling which has important role in cardiovascular physiology and pathophysiology [35]. It is suggested mutation in the DTNA gene may have disturb the interaction of the gene at the protein level and indirectly effect the Rho signaling pathway. Although more functional studies are required to delve the exact mechanism of this mutation on the function of DTNA protein.

Previous studies have demonstrated that the majority of mutations (both loss-of-function and gain-of-function types) related to $\mathrm{AF}$ are harbored in the genes encoding ion channel subunits $[6,15,21-23]$. However, diseasecausing mutations in non-ion channel coding genes have also been reported [18-20]. In line with the latter one, in the present study, the identified mutation was located in a non-ion channel coding gene. Therefore, many more molecular genetic studies are needed to clarify new genetic alterations and validate genotype-phenotype associations with a view to shedding light on the pathophysiological pathways of AF. 


\section{Limitations}

The results of the present study should be interpreted in light of certain limitations. Functional genomic studies were not carried out in the present study. In addition, experimental studies and expression analyses are essential to determine the exact role of this variant responsible for AF. The transition was not assessed in the proband's uncle, who suffered from AF, and nor was it evaluated in the other apparently normal family members who were not available for the study.

\section{Conclusions}

The results of the current study present the first evidence for the association between a rare missense variant in DTNA and early-onset AF. Not only do these findings expand our knowledge regarding the genetics of AF but also they may have further implications for the treatment and prevention of AF. Although the discovery of novel AF-associated genetic variants has gained momentum in recent years, there is a paucity of information on the genetic analysis of AF in the Iranian population. The genetic basis of AF in the Iranian population undoubtedly needs further in-depth research.

\section{Abbreviations}

AF: Atrial fibrillation; DTNA: Human a-dystrobrevin gene; PCR: Polymerase chain reaction; Oligos: Oligonucleotides; NEBL: Nebulette; SCN5A: Sodium voltage-gated channel a subunit 5; LVNC: Left ventricular noncompaction cardiomyopathy; DCM: Dilated cardiomyopathy; HCM: Hypertrophic cardiomyopathy; MD: Meniere's disease.

\section{Supplementary Information}

The online version contains supplementary material available at https://doi. org/10.1186/s12872-022-02485-0.

Additional file 1: Table S1. Sequences of the oligos utilized for polymerase chain reaction and Sanger sequencing. Table S2. Clinical information of all the available members of the pedigree. Table S3. All the nucleotide variations in the ClinVar Database that are submitted as pathogenic and the ones with conflicting interpretations concerning pathogenicity. As is shown in the table, except for 2 variations, c.146A $>\mathrm{G}$ and c.362C $>\mathrm{T}$, which were causative for left ventricular noncompaction cardiomyopathy, all the other transitions have not been determined as causative variations for diseases yet. LVNC: Left Ventricular Noncompaction Cardiomyopathy, DCM: Dilated Cardiomyopathy, HCM: Hypertrophic Cardiomyopathy, MD: Meniere's disease.

\section{Acknowledgements}

We wish to thank the patient and his family for their kind participation in the study. We are grateful to Mrs. Nooshin Ashrafi for her technical assistance.

\section{Authors' contributions}

MM: experiment design, data production, data interpretation, writing the first draft of MS, and manuscript final edit. MJ: lab work, data production, and MS edit. SK: WES analysis and MS edit. MH: lab work, data production, and MS edit. $\mathrm{MH}$ : clinical evaluation and MS edit. $\mathrm{MH}$ : experiment design, clinical evaluation of patients, data production, data interpretation, and manuscript final edit. All the authors contributed to the article and approved the submitted version. All authors read and approved the final manuscript.

\section{Funding}

This work was supported in part by a research grant to Dr. Haghjoo from The National Institute for Medical Research Development (NIMAD, 971510) and the Research Deputyship of Rajaie Cardiovascular Medical and Research Center (9774).

\section{Availability of data and materials}

Accession number The accession number of the reported variant in paper is available in clinVAR repository with the VCV001224303 accession number. The data sets presented in the present study can be reached in online repositories. The identified nucleotide transitions were analyzed through the UCSC Genome Browser (https://genome.ucsc.edu) and ClinVar (www.ncbi.nlm.nih. gov/clinvar) databases. In silico predictive software tools such as SIFT (https:// sift.bii.a-star.edu.sg), PROVEAN (provean.jcvi.org), PolyPhen-2 (genetics.bwh. harvard.edu/pph2), and MutationTaster (www.mutationtaster.org) were applied to study the pathogenesis of the detected nucleotide variations. The Protein Homology/analogY Recognition Engine V 2.0 (Phyre2) and DNASTAR (https://www.dnastar.com) bioinformatics software tools were utilized to predict the secondary and third structures of the DTNA wild type and mutant protein. The accession number and all the repositories used for the study are mentioned in the article as well.

\section{Declarations}

\section{Ethics approval and consent to participate}

The study protocol was approved by the National Institute for Medical Research Development (NIMAD, 971510) and the Ethics Committee of Rajaie Cardiovascular Medical and Research Center (RHC.AC.IR.REC.1397.088). The study was conducted in accordance with the Helsinki Declaration. All the individuals who joined the study signed written informed consent.

\section{Consent for publication}

Not applicable.

\section{Competing interests}

The authors hereby declare no competing interest.

\section{Author details}

${ }^{1}$ Cardiogenetic Research Center, Rajaie Cardiovascular Medical and Research Center, Iran University of Medical Sciences, Tehran, Iran. ${ }^{2}$ Cardiac Electrophysiology Center, Rajaie Cardiovascular Medical and Research Center, Iran University of Medical Sciences, Vali-Asr St, Hashemi-Rafsanjani Blvd, Tehran, Iran.

Received: 27 October 2021 Accepted: 31 January 2022

Published online: 11 February 2022

\section{References}

1. Kornej J, Börschel CS, Benjamin EJ, Schnabel RB. Epidemiology of atrial fibrillation in the 21st century: novel methods and new insights. Circ Res. 2020;127(1):4-20.

2. Schnabel RB, Yin X, Gona P, Larson MG, Beiser AS, McManus DD, et al. 50 year trends in atrial fibrillation prevalence, incidence, risk factors, and mortality in the Framingham Heart Study: a cohort study. The Lancet. 2015;386(9989):154-62.

3. Huxley RR, Lopez FL, Folsom AR, Agarwal SK, Loehr LR, Soliman EZ, et al. Absolute and attributable risks of atrial fibrillation in relation to optimal and borderline risk factors: the Atherosclerosis Risk in Communities (ARIC) study. Circulation. 2011;123(14):1501-8.

4. Chugh SS, Havmoeller R, Narayanan K, Singh D, Rienstra M, Benjamin EJ, et al. Worldwide epidemiology of atrial fibrillation: a global burden of disease 2010 Study. Circulation. 2014;129(8):837-47.

5. Roberts JD, Gollob MH. A contemporary review on the genetic basis of atrial fibrillation. Methodist Debakey Cardiovasc J. 2014;10(1):18. 
6. Roselli C, Chaffin MD, Weng L-C, Aeschbacher S, Ahlberg G, Albert CM, et al. Multi-ethnic genome-wide association study for atrial fibrillation. Nat Genet. 2018:50(9):1225-33.

7. Heidarali M, Bakhshandeh H, Fazelifar A, Haghjoo M. Clinical profile and outcome of familial versus non-familial atrial fibrillation. Int J Cardiol. 2020;314:70-4.

8. Fox CS, Parise H, D'Agostino RB Sr, Lloyd-Jones DM, Vasan RS, Wang TJ, et al. Parental atrial fibrillation as a risk factor for atrial fibrillation in offspring. JAMA. 2004;291(23):2851-5.

9. Chang S-H, Kuo C-F, Chou I-J, See L-C, Yu K-H, Luo S-F, et al. Association of a family history of atrial fibrillation with incidence and outcomes of atrial fibrillation: a population-based family cohort study. JAMA Cardiol. 2017;2(8):863-70

10. Gundlund A, Fosbøl EL, Kim S, Fonarow GC, Gersh BJ, Kowey PR, et al. Family history of atrial fibrillation is associated with earlier-onset and more symptomatic atrial fibrillation: results from the Outcomes Registry for Better Informed Treatment of Atrial Fibrillation (ORBIT-AF) registry. Am Heart J. 2016;175:28-35.

11. Christophersen IE, Ravn LS, Budtz-Joergensen E, Skytthe A, Haunsoe S, Svendsen $\mathrm{JH}$, et al. Familial aggregation of atrial fibrillation: a study in Danish twins. Circ Arrhythm Electrophysiol. 2009;2(4):378-83.

12. Lubitz SA, Yin X, Fontes JD, Magnani JW, Rienstra M, Pai M, et al. Association between familial atrial fibrillation and risk of new-onset atrial fibrillation. JAMA. 2010;304(20):2263-9.

13. Heidarali M, Bakhshandeh H, Golpira R, Fazelifar A, Alizadeh-Diz A, Emkanjoo Z, et al. A prospective survey of atrial fibrillation management in Iran: baseline results of the Iranian Registry of Atrial Fibrillation (IRAF). Int J Clin Pract. 2021;75:e14313.

14. Andersen $\mathrm{JH}$, Andreasen $\mathrm{L}$, Olesen MS. Atrial fibrillation-a complex polygenetic disease. Eur J Human Genet. 2021;29(7):1051-60. https://doi. org/10.1038/s41431-020-00784-8.

15. Ragab AA, Sitorus GD, Brundel BB, Groot N. The genetic puzzle of familial atrial fibrillation. Front Cardiovasc Med. 2020;7:14

16. Bai D. Atrial fibrillation-linked GJA5/connexin40 mutants impaired gap junctions via different mechanisms. FEBS Lett. 2014;588(8):1238-43.

17. Hodgson-Zingman DM, Karst ML, Zingman LV, Heublein DM, Darbar D, Herron KJ, et al. Atrial natriuretic peptide frameshift mutation in familial atrial fibrillation. N Engl J Med. 2008:359(2):158-65.

18. Huang R-T, Xue S, Xu Y-J, Zhou M, Yang Y-Q. A novel NKX2. 5 loss-offunction mutation responsible for familial atrial fibrillation. Int J Mol Med. 2013;31(5):1119-26.

19. Xiang R, Fan L-L, Huang H, Cao B-B, Li X-P, Peng D-Q, et al. A novel mutation of GATA4 (K319E) is responsible for familial atrial septal defect and pulmonary valve stenosis. Gene. 2014;534(2):320-3.

20. Brauch KM, Chen LY, Olson TM. Comprehensive mutation scanning of LMNA in 268 patients with lone atrial fibrillation. Am J Cardiol. 2009;103(10):1426-8.

21. Roselli C, Rienstra M, Ellinor PT. Genetics of atrial fibrillation in 2020: GWAS, genome sequencing, polygenic risk, and beyond. Circ Res. 2020;127(1):21-33.

22. Darbar D, Kannankeril PJ, Donahue BS, Kucera G, Stubblefield T, Haines $J$, et al. Cardiac sodium channel (SCN5A) variants associated with atrial fibrillation. Circulation. 2008;117(15):1927-35.

23. Ellinor PT, Petrov-Kondratov VI, Zakharova E, Nam EG, MacRae CA. Potassium channel gene mutations rarely cause atrial fibrillation. BMC Med Genet. 2006;7(1):1-5.

24. Bozkurt B, Colvin M, Cook J, Cooper L, Deswal A, Fonarow G, et al. American Heart Association Committee on Heart Failure and Transplantation of the Council on Clinical Cardiology; Council on Cardiovascular Disease in the Young; Council on Cardiovascular and Stroke Nursing; Council on Epidemiology and Prevention; and Council on Quality of Care and Outcomes Research. Current diagnostic and treatment strategies for specific dilated cardiomyopathies: a scientific statement from the American Heart Association. Circulation. 2016:134(23):e579-646.

25. Kelley LA, Mezulis S, Yates CM, Wass MN, Sternberg MJ. The Phyre2 web portal for protein modeling, prediction and analysis. Nat Protoc. 2015;10(6):845-58.

26. Langmead B, Trapnell C, Pop M, Salzberg SL. Ultrafast and memoryefficient alignment of short DNA sequences to the human genome Genome Biol. 2009;10(3):R25.
27. Garrison E, Marth G. Haplotype-based variant detection from short-read sequencing. arXiv preprint arXiv:1207.3907. 2012.

28. Fatkin D, Santiago CF, Huttner IG, Lubitz SA, Ellinor PT. Genetics of atrial fibrillation: state of the art in 2017. Heart Lung Circ. 2017;26(9):894-901.

29. Olesen MS, Nielsen MW, Haunsø S, Svendsen JH. Atrial fibrillation: the role of common and rare genetic variants. Eur J Hum Genet. 2014:22(3):297-306.

30. Sadoulet-Puccio HM, Feener CA, Schaid DJ, Thibodeau SN, Michels WV, Kunkel LM. The genomic organization of human dystrobrevin. Neurogenetics. 1997; 1(1):37-42

31. Cao Q, Shen Y, Liu X, Yu X, Yuan P, Wan R, et al. Phenotype and functional analyses in a transgenic mouse model of left ventricular noncompaction caused by a DTNA mutation. Int Heart J. 2017;58:939-47.

32. Ichida F, Tsubata S, Bowles KR, Haneda N, Uese K, Miyawaki T, et al. Novel gene mutations in patients with left ventricular noncompaction or Barth syndrome. Circulation. 2001;103(9):1256-63.

33. Requena T, Cabrera S, Martín-Sierra C, Price SD, Lysakowski A, LopezEscamez JA. Identification of two novel mutations in FAM136A and DTNA genes in autosomal-dominant familial Meniere's disease. Hum Mol Genet. 2015:24(4):1119-26.

34. Sadoulet-Puccio HM, Khurana TS, Cohen JB, Kunkel LM. Cloning and characterization of the human homologue of a dystrophin related phosphoprotein found at the Torpedo electric organ post-synaptic membrane. Hum Mol Genet. 1996:5(4):489-96.

35. Loirand G, Guérin P, Pacaud P. Rho kinases in cardiovascular physiology and pathophysiology. Circ Res. 2006;98(3):322-34.

\section{Publisher's Note}

Springer Nature remains neutral with regard to jurisdictional claims in published maps and institutional affiliations.

Ready to submit your research? Choose BMC and benefit from

- fast, convenient online submission

- thorough peer review by experienced researchers in your field

- rapid publication on acceptance

- support for research data, including large and complex data types

- gold Open Access which fosters wider collaboration and increased citations

- maximum visibility for your research: over 100M website views per year

At BMC, research is always in progress.

Learn more biomedcentral.com/submissions 